\title{
Sound propagation and oscillations of a superfluid Fermi gas in the presence of a 1D optical lattice
}

\author{
L.P. Pitaevskii, ${ }^{1,2}$ S. Stringari, ${ }^{1}$ and G. Orso $^{1}$ \\ ${ }^{1}$ Dipartimento di Fisica, Universita' di Trento and BEC-INFM, 1-38050 Povo, Italy \\ ${ }^{2}$ Kapitza Institute for Physical Problems, 117334 Moscow, Russia
}

(Dated: October 25, 2018)

\begin{abstract}
We develop the hydrodynamic theory of Fermi superfluids in the presence of a periodic potential. The relevant parameters governing the propagation of sound (compressibility and effective mass) are calculated in the weakly interacting BCS limit. The conditions of stability of the superfluid motion with respect to creation of elementary excitations are discussed. We also evaluate the frequency of the center of mass oscillation when the superfluid gas is additionally confined by a harmonic trap.
\end{abstract}

PACS numbers:

It is well known that sound cannot propagate in a degenerate noninteracting Fermi gas. In fact the sound velocity $c=v_{F} / \sqrt{3}$, calculated from the compressibility of the gas, is smaller than the Fermi velocity $v_{F}$, so that sound is unstable towards decay into the continuum of particle-hole excitations. In the presence of an attractive interaction, Cooper pairing opens a gap at the Fermi level. As a result, sound waves with sufficiently low frequency can propagate in a neutral superfluid. An important question is to understand how these modes behave when the system is confined by a periodic potential.

The propagation of sound in Bose gases trapped by an optical lattice has been already the object of extensive theoretical work [1]. Experiments [2] on the collective oscillations have confirmed that the low energy dynamic behaviour of a Bose-Einstein condensed gas is well described by the hydrodynamic equations of superfluids [3], where the coherent tunneling through the barriers generated by the periodic potential is taken into account by a proper renormalization of the effective mass.

In this paper we generalize the hydrodynamic equations of Fermi superfluids in order to investigate the propagation of sound and the collective oscillations of a Fermi gas in the presence of a $1 \mathrm{D}$ periodic potential generated by a laser field.

We consider an interacting two-component atomic Fermi gas trapped by a potential $V_{\text {ext }}$ given by the sum of a 3D harmonic trap and of a stationary 1D optical potential modulated along the $z$-axis:

$$
V_{e x t}=V_{h o}+s E_{R} \sin ^{2} q_{B} z
$$

where $V_{h o}=m\left(\omega_{\perp}^{2} r_{\perp}^{2}+\omega_{z}^{2} z^{2}\right) / 2$ and $E_{R}=\hbar^{2} q_{B}^{2} / 2 m$ is the recoil energy. Here $q_{B}$ is the Bragg momentum and $s$ is a dimensionless parameter providing the intensity of the laser beam. The optical potential has periodicity $d=\pi / q_{B}$ along the $z$-axis.

The long wavelength dynamic behaviour of a neutral superfluid is properly described by Landau's hydrodynamic theory. At $T=0$ the relevant variables are the density $n$ of particles and the superfluid velocity

$$
\mathbf{v}=\frac{\hbar}{2 m} \nabla \phi
$$

where $m$ is the atomic mass and $\phi$ is the phase of the order parameter defined through the anomalous mean value $\left\langle\Psi_{\uparrow}(\mathbf{r}) \Psi_{\downarrow}(\mathbf{r})\right\rangle=\left|\left\langle\Psi_{\uparrow}(\mathbf{r}) \Psi_{\downarrow}(\mathbf{r})\right\rangle\right| e^{i \phi(\mathbf{r})}$.

The hydrodynamic equations can be derived from a variational principle, starting from an effective action describing the low energy collective excitations over the ground state. In the weak coupling limit these excitations are known as the Bogolubov-Anderson modes. The Lagrangian of the system takes the general form

$$
L=\int d \mathbf{r}\left[e(n, \mathbf{v})+V_{h_{o}} n+n \frac{\partial}{\partial t} \frac{\phi}{2}\right]
$$

where $e(n, \mathbf{v})$ is the energy per unit of volume. By expanding $e(n, \mathbf{v})$ up to the terms quadratic in $\mathbf{v}$ one finds

$$
e(n, \mathbf{v})=e(n, 0)+\frac{1}{2} n \mathbf{v}_{\perp}^{2}+\frac{1}{2} \frac{m^{2}}{\tilde{m}} n v_{z}^{2}
$$

where $\tilde{m}(\geq m)$ is an effective mass accounting for the increased inertia of the superfluid along the direction of the laser. In general $\tilde{m}$ is density dependent. In Eq.(3) we have assumed that the two spin species are equally populated $\left(n=2 n_{\uparrow}\right)$. Inserting Eq.(4) in the Lagrangian (3) yields the hydrodynamic equations

$$
\frac{\partial n}{\partial t}+\partial_{x}\left(n v_{x}\right)+\partial_{y}\left(n v_{y}\right)+\partial_{z}\left(\frac{m}{\tilde{m}} n v_{z}\right)=0,
$$

$\frac{\partial}{\partial t} \mathbf{v}+\frac{1}{m} \nabla\left(\mu(n)+V_{h o}+\frac{m}{2} v_{x}^{2}+\frac{m}{2} v_{y}^{2}+\frac{\partial}{\partial n}\left(\frac{m}{\tilde{m}} n\right) \frac{m}{2} v_{z}^{2}\right)=0$,

where $\mu(n)=\partial e / \partial n$ is the chemical potential. Generalization to include $2 \mathrm{D}$ or $3 \mathrm{D}$ optical lattices is trivial.

Let us first consider the propagation of sound in the absence of the harmonic potential $\left(V_{h o}=0\right)$. We will include later the harmonic trap to investigate the oscillations of the cloud. If $V_{h o}=0$ Eqs. (5) and (6) admit 
linearized solutions around the equilibrium density $n_{0}$ of the form $n=n_{0}+\delta n$, with $\delta n \sim e^{i q z-i \omega t}$ and analogously for $v_{z}$. This permits to derive the phonon dispersion $\omega=c_{z} q$ along the direction of the optical lattice, with the sound velocity given by

$$
c_{z}=\sqrt{\frac{n}{\tilde{m}} \frac{\partial \mu}{\partial n}} .
$$

The presence of the periodic lattice enters Eq.(7) through both the renormalization of $\tilde{m}$ and the change of the inverse compressibility $n \partial \mu / \partial n$. Analogously, the sound velocity along the transverse directions, where the gas is free, is given by $c_{\perp}^{2}=(n / m) \partial \mu / \partial n$.

Equations (5 7) are very general and apply also to strongly interacting superfluids. They permit to calculate the low energy dynamics of the system once the equation of state and the effective mass are known. In the following we will apply these equations to a dilute Fermi gas interacting with negative scattering length (BCS limit). In this case the internal energy is fixed by the quantum pressure and therefore the chemical potential is equal to the Fermi energy $\left(\mu=\epsilon_{F}\right)$. For simplicity we consider values of the Fermi energy such that only the lowest Bloch band is populated at zero temperature. The single particle energy spectrum can then be written as $\epsilon(\mathbf{k})=\mathbf{k}_{\perp}^{2} / 2 m+\epsilon\left(k_{z}\right)$ where $\epsilon\left(k_{z}\right)$ is the dispersion relation of the lowest Bloch band. The energy of the system at rest is given by $E=V \int \epsilon(\mathbf{k}) n_{\mathbf{k}} d \mathbf{k} /(2 \pi \hbar)^{3}$, where $n_{\mathbf{k}}=2 \Theta\left(\epsilon_{F}-\epsilon(\mathbf{k})\right)$ is the quasi-momentum distribution of the non-interacting gas [4], $\Theta(x)$ being the usual step function, $V$ is the volume of the system and the integration over $k_{z}$ is restricted to the first Brillouin zone $-\pi \hbar / d \leq k_{z}<\pi \hbar / d$. The Fermi energy $\epsilon_{F}$ is related to the atomic density by the normalization condition $n=\int n_{\mathbf{k}} d \mathbf{k} /(2 \pi \hbar)^{3}$.

Let us first assume that the gas moves with constant superfluid velocity $\mathbf{v}$. The order parameter acquires the phase $\phi=2 m \mathbf{v} \cdot \mathbf{r} / \hbar$ (see Eq.(2)) and, correspondingly, the field operator $\hat{\Psi}_{\sigma}$ transforms according to

$$
\hat{\Psi}_{\sigma}(\mathbf{r}) \rightarrow \hat{\Psi}_{\sigma}(\mathbf{r}) e^{i m \mathbf{v} \cdot \mathbf{r} / \hbar}
$$

Equation (8) is a gauge transformation which does not change the energy levels, but changes their classification. In particular the Bloch state of quasi-momentum $\mathbf{k}$ is mapped into the state $\mathbf{k}+m \mathbf{v}$ (and hence $\epsilon(\mathbf{k})$ into $\epsilon(\mathbf{k}+m \mathbf{v}))$. The energy density of the moving system can therefore be written as:

$$
e(n, \mathbf{v})=\int \epsilon(\mathbf{k}+m \mathbf{v}) n_{\mathbf{k}} \frac{d \mathbf{k}}{(2 \pi \hbar)^{3}}
$$

where the density dependence comes from the quasimomentum distribution $n_{\mathbf{k}}$. We can now expand Eq. (9) in powers of $\mathbf{v}$. The linear term vanishes by symmetry as required by the stability of the ground state. The first non vanishing contribution is the quadratic, diamagnetic term. Comparison with Eq.(4) then permits to find, after an integration by parts, the useful expression

$$
\frac{1}{\tilde{m}}=\frac{\int\left(\partial \epsilon / \partial k_{z}\right)^{2} \delta\left(\epsilon_{F}-\epsilon(\mathbf{k})\right) d \mathbf{k} /(2 \pi \hbar)^{3}}{n / 2}
$$

for the effective mass, holding for a dilute superfluid Fermi gas at zero temperature. From Eqs (92) and (10), we also see that the current density along $z$ in the equation of continuity (5) corresponds to $j_{z}=$ $(1 / m V) \delta E / \delta v_{z}$.

From Eq.(10) we see that only the states near the Fermi surface contribute to the effective mass. In general $\tilde{m}$ depends on the Fermi energy or, equivalently, on the density. An important exception occurs when the Bloch band is almost empty. In this case the occupied states obey an approximate quadratic dispersion $\epsilon\left(k_{z}\right)=k_{z}^{2} / 2 m^{*}$, where $m^{*}$ depends only on the laser intensity. From Eq.(10) we then find $\tilde{m}=m^{*}$. This coincides with the result for the effective mass holding for a dilute Bose-Einstein condensed gas in the same optical lattice [5]. Equation (10) also shows that the integration along the transverse directions plays a crucial role in keeping $\tilde{m}$ finite as the Fermi energy crosses the Bloch bandwith. In fact if we neglect the radial dispersion and consider a pure 1D system, the effective mass (10) would actually diverge as the band is completely filled.

A second crucial ingredient needed to calculate the velocity of sound (see Eq.(17)) is the compressibility $(n \partial \mu / \partial n)^{-1}$. In a dilute Fermi gas at zero temperature this is simply calculated in terms of the density of states as

$$
\left(n \frac{\partial \mu}{\partial n}\right)^{-1}=\frac{\int \delta\left(\epsilon_{F}-\epsilon(\mathbf{k})\right) d \mathbf{k} /(2 \pi \hbar)^{3}}{n / 2} .
$$

The optical lattice modifies the compressibility with respect to the value $\left(3 \pi^{2}\right)^{2 / 3} \hbar^{2} n^{2 / 3} / 3 m$ holding for a uniform gas. By inserting Eqs (10) and (11) into Eq.(7), we find the result

$$
c_{z}^{2}=\frac{\int\left(\partial \epsilon / \partial k_{z}\right)^{2} \delta\left(\epsilon_{F}-\epsilon(\mathbf{k})\right) d \mathbf{k}}{\int \delta\left(\epsilon_{F}-\epsilon(\mathbf{k})\right) d \mathbf{k}}
$$

for $c_{z}^{2}$, which can be regarded as the average of the square of the group velocity $\partial \epsilon / \partial k_{z}$ over the Fermi surface. Again it should be noted that, due to the free dispersion in the transverse direction, the sound velocity (12) remains finite as $\epsilon_{F}$ crosses the bandwidth. In a pure $1 \mathrm{D}$ system, Eq.(12) would predict $c_{z}=0$ when the band is completely filled, since $\partial \epsilon / \partial k_{z}$ vanishes at the edge of the Brillouin zone.

Let us point out that Eq. (12) has been derived starting from the hydrodynamic equations but the same result can be obtained by generalizing BCS theory through the inclusion of the interaction between Bogolubov quasiparticles. This can be achieved, for example, using time 
dependent Hartree-Fock theory [ 6$]$ or the Random Phase Approximation (RPA) 7].

If the laser intensity is sufficiently large one can work in the tight-binding approximation where the dispersion of the lowest Bloch band takes the simple form $\epsilon\left(k_{z}\right)=$ $\delta\left(1-\cos \left(k_{z} d / \hbar\right)\right)$, with $\delta$ proportional to the tunneling rate between two consecutive wells. In this case the equation of state can be calculated analytically. For values of the Fermi energy above the bandwidth $\left(\epsilon_{F}>2 \delta\right)$ one finds $n=m\left(\epsilon_{F}-\delta\right) / \pi \hbar^{2} d$ and hence $d n / d \mu=m / \pi \hbar^{2} d$. For smaller values $\left(\epsilon_{F} \leq 2 \delta\right)$ one instead finds $n=$ $(2 \sqrt{y(1-y)}+(2 y-1) \arccos (1-2 y)) m \delta / \pi^{2} \hbar^{2} d$, yielding $d n / d \mu=\left(m / \pi^{2} \hbar^{2} d\right) \arccos (1-2 y)$, where $y=\epsilon_{F} / 2 \delta$.

The effective mass (10) increases as the density of particles increases. For values of $\epsilon_{F}<2 \delta$ one finds $\tilde{m}=$ $\left(2 \pi^{2} \hbar^{4} n / \delta^{2} d m\right)[2(2 y-1) \sqrt{y(1-y)}+\arccos (1-2 y)]^{-1}$ and in the limit $y \ll 1$, corresponding to $\epsilon_{F} \ll 2 \delta$, $\tilde{m}$ approaches the density independent value $m^{*}=\hbar^{2} / \delta d^{2}$. Conversely, for $\epsilon_{F}>2 \delta$ the effective mass scales linearly with the density: $\tilde{m}=2 \hbar^{4} \pi n / m \delta^{2} d$. This result can be obtained in a BCS approach, following Ref. [8].

Let us now use Eq.(7) to evaluate the behaviour of the sound velocity along the direction of the optical confinement. In the limit $\epsilon_{F}>2 \delta$ one finds the density independent value $c_{z}^{2}=\delta^{2} d^{2} / 2 \hbar^{2}$. This is simply understood by noticing that $\partial \epsilon / \partial k_{z}=(\delta d / \hbar) \sin \left(k_{z} d / \hbar\right)$ and that the average of $\sin ^{2}\left(k_{z} d / \hbar\right)$ over the first Brillouin zone gives the factor $1 / 2$. For $\epsilon_{F}<2 \delta$, the longitudinal sound velocity is related to the Fermi energy by the expression

$$
c_{z}^{2}=\left(\frac{\delta d}{\hbar}\right)^{2}\left[\frac{(2 y-1) \sqrt{y(1-y)}}{\arccos (1-2 y)}+\frac{1}{2}\right] .
$$

In particular, for $y \ll 1$, corresponding to $\epsilon_{F} \ll 2 \delta$, Eq. (13) reduces to $c_{z}^{2}=2 \epsilon_{F} / 3 m^{*}$. It is important to notice that the continuous hydrodynamic approach along the direction of the laser field requires that the phase $\phi$ of the order parameter varies slowly between neighboring wells. Taking into account that the energy of the phonons must be small compared to the superfluid gap $\Delta$, we see that our theory is valid provided $q_{z} \ll \min \left(\Delta / \hbar c_{z}, d^{-1}\right)$ where $q_{z}$ is the wave vector of the sound excitation.

The propagation of sound in the transverse direction is affected by the presence of the optical lattice only through the change of the compressibility. For values of the density corresponding to $\epsilon_{F}>2 \delta$, one finds $c_{\perp}^{2}=\left(\epsilon_{F}-\delta\right) / m$. For smaller values of $\epsilon_{F}$ one finds $c_{\perp}^{\frac{1}{2}}=\pi^{2} \hbar^{2} d n / m^{2} \arccos (1-2 y)$, yielding $c_{\perp}^{2}=2 \epsilon_{F} / 3 m$ in the limit $\epsilon_{F} \ll 2 \delta$. In the case of transverse sound propagation, the hydrodynamic theory is valid under the usual assumptions $q_{\perp} \ll \Delta / \hbar c_{\perp}$.

The hydrodynamic equations (56) can be generalized to include finite velocity of the gas with respect to the optical lattice. This is important in order to study the conditions of stability of the moving fluid with respect to the creation of elementary excitations. This problem has been recently addressed in the case of Bose-Einstein condensates, both from the theoretical [9] and experimental [10] point of view.

Starting from the general form (3) for the Lagrangian and setting $V_{h o}=0$, one finds

$$
\begin{aligned}
\frac{\partial n}{\partial t}+\nabla \mathbf{j}(n, \mathbf{v}) & =0 \\
m \frac{\partial \mathbf{v}}{\partial t}+\nabla \mu(n, \mathbf{v}) & =0
\end{aligned}
$$

where $\mathbf{j}=(1 / m) \partial e / \partial \mathbf{v}, \mu=\partial e / \partial n$ are, respectively, the current and the chemical potential of the gas while $e(n, \mathbf{v})$ is defined in Eq. (9).

Linearizing Eq.s (1415) with $n(\mathbf{r})=n_{0}+\delta n(z)$ and $\mathbf{v}(\mathbf{r})=(k / m+\delta v(z)) \hat{\mathbf{z}}$ and looking for solutions of the form $\delta n, \delta v \sim e^{i(q z-\omega t)}$, we find the dispersion relation

$$
\omega=\frac{\partial^{2} \epsilon}{\partial n \partial k} q+\sqrt{\frac{\partial^{2} \epsilon}{\partial n^{2}} \frac{\partial^{2} \epsilon}{\partial k^{2}}}|q| .
$$

Dynamical instability occurs when the argument in the square root of Eq. (16) becomes negative. This leads to complex frequency and corresponds to an exponential growth of excitations wich destabilize the superfluid. Energetic instability instead takes place when $\omega$ becomes negative, i.e. when the first term in Eq. (16) (accounting for the Doppler shift) is in modulus larger than the second one.

In tight-binding limit the energy density (9) takes the simple form

$$
\epsilon(n, k)=\epsilon(n, 0)+\frac{n}{\tilde{m}}\left(\frac{\hbar}{d}\right)^{2}\left(1-\cos \left[\frac{k d}{\hbar}\right]\right)
$$

where $\tilde{m}$ is defined in Eq.(10). Note that in the limit of small $k$ Eq.(17) reduces to Eq.(4) with $\mathbf{v}_{\perp}=0$. By inserting Eq.(17) into Eq.(16) we find

$$
\omega=\frac{\partial}{\partial n}\left(\frac{n}{\tilde{m}}\right) \frac{\hbar}{d} \sin \left[\frac{k d}{\hbar}\right] q+\sqrt{\frac{\partial^{2} \epsilon}{\partial n^{2}} \frac{n}{\tilde{m}} \cos \left[\frac{k d}{\hbar}\right]}|q| .
$$

We see from Eq.(18) that in tight-binding limit the instability opens at $k=\pi \hbar / 2 d$ similar to what happens in Bose-Einstein condensates [9]. One should notice that results (17/18), being derived from hydrodynamic theory, holds only for small values of $q$ 11. There are however no restrictions on the value of the stationary velocity $\mathrm{k} / \mathrm{m}$ of the fluid with respect to the lattice.

For $\epsilon_{F} \ll 2 \delta$, where $\tilde{m}=m^{*}$, the Doppler term is exactly the same as for Bose condensates. For higher values of the density, however, this term becomes smaller and vanishes identically for $\epsilon_{F}>2 \delta$, where $\tilde{m}$ is linear in the density $n$. In this limit the current becomes density independent while the chemical potential no longer depends on the velocity of the fluid.

So far we have discussed the behaviour of a superfluid gas confined by a periodic potential. In the presence of 
the additional harmonic potential $V_{h o}$ the low energy oscillations exhibit new features. Of particular importance is the center of mass oscillation along the direction of the optical lattice. In the absence of the periodic potential, this mode would oscillate exactly at the frequency of the harmonic trap. The presence of the periodic potential gives rise to new interesting features. In a recent work 12 it has been shown that in a non-interacting Fermi gas with Fermi energy $\epsilon_{F}$ larger than the Bloch bandwith $2 \delta$, the center of mass cannot oscillate around the bottom of the trap, but remains localized at one side of the harmonic field. Inclusion of collisions in a two-component gas favours the relaxation towards the equilibrium configuration. However, collisions cannot restore the propagation of sound in the hydrodynamic regime as happens in the absence of the periodic potential. In fact, it was concluded in Ref. 13. that, under the condition $\epsilon_{F}>2 \delta$, the center of mass oscillations of a non superfluid gas are overdamped in the collisional regime, due to umklapp processes.

As recently suggested by Wouters et al. [14], in the superfluid regime the center of mass can instead oscillate and the corresponding frequency can be calculated starting from the hydrodynamic equations (5) and (6). We will make the natural ansatz

$$
\phi=\alpha(t) z, \quad n=n_{0}\left(z-a(t), \mathbf{r}_{\perp}\right),
$$

for the phase and for the density distribution where $\alpha$ and $a$ are functions of time and $n_{0}(\mathbf{r})=2 \int \Theta\left(\epsilon_{F}(\mathbf{r})-\right.$ $\epsilon(\mathbf{k})) d \mathbf{k} /(2 \pi \hbar)^{3}$ is the equilibrium density evaluated in the local density approximation with $\epsilon_{F}(\mathbf{r})=\epsilon_{F}-V_{h o}(\mathbf{r})$. This ansatz corresponds to a rigid shift of the density in coordinate space accompained by a uniform velocity field $v_{z}=\hbar \alpha / 2 m$. By inserting the ansatz (19) into the Lagrangian (31) and retaining only the lowest order (quadratic) terms in the functions $a$ and $\alpha$, one can recast (3i) in the form $L(\alpha, \dot{\alpha}, a) \propto m \omega_{z}^{2} a^{2} / 2+\alpha^{2} / 8 m_{C M}+a \dot{\alpha} / 2$, where we have introduced the averaged effective mass 15 .

$$
\begin{array}{r}
\frac{1}{m_{C M}}=\frac{1}{N} \int \frac{1}{\tilde{m}} n_{0}(\mathbf{r}) d \mathbf{r} \\
=\frac{2}{N} \int\left(\partial \epsilon / \partial k_{z}\right)^{2} \delta\left(\epsilon_{F}(\mathbf{r})-\epsilon(\mathbf{k})\right) d \mathbf{k} d \mathbf{r} /(2 \pi \hbar)^{3}
\end{array}
$$

and $N=\int n_{0}(\mathbf{r}) d \mathbf{r}$ is the total number of particles. The above Lagrangian describes a simple harmonic oscillator with frequency

$$
\omega_{C M}=\omega_{z} \sqrt{\frac{m}{m_{C M}}} .
$$

Result (21) for the frequency of the center of mass oscillation can be also derived microscopically using a sum rule approach, based on the ratio

$$
(\hbar \omega)^{2}=\frac{m_{1}}{m_{-1}}
$$

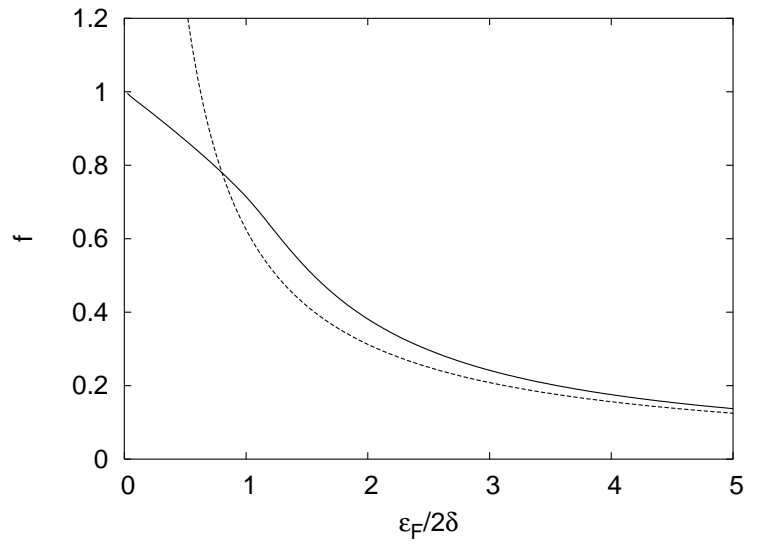

FIG. 1: Function $f$ [See Eq.(23)] versus the parameter $y=$ $\epsilon_{F} / 2 \delta$ (solid line). The asymptotic limit $5 / 8 y$ is also shown (dashed line).

between the energy weighted and inverse energy weighted moments [5] relative to the dipole operator $D=\sum_{j=1}^{N} z_{j}$. The energy weighted moment can be calculated using the general expression $m_{1}=(1 / 2)\langle[D,[H, D]]\rangle$ where \langle\rangle is the average over the ground state and the effective Hamiltonian $H=\sum_{j} \epsilon\left(\mathbf{k}_{j}\right)+\sum_{j} V_{h o}\left(\mathbf{r}_{j}\right)+\sum_{j<\ell} V_{2-\text { body }}\left(\mid \mathbf{r}_{k}-\right.$ $\left.\mathbf{r}_{\ell} \mid\right)$ is the projection of the full Hamiltonian into the lowest Bloch band. One finds $m_{1}=(1 / 2)\left\langle\sum_{j=1}^{N} \partial^{2} \epsilon / \partial k_{z_{j}}^{2}\right\rangle$, which is the analog of the f-sum rule [16]. On the other hand the inverse energy weighted moment is easily related to the dipole static polarizability $\alpha_{D}$, fixed by the harmonic potential: $m_{-1}=(1 / 2) \alpha_{D}=N /\left(2 m \omega_{z}^{2}\right)$. By evaluating the average $\left\langle\sum_{j=1}^{N} \partial^{2} \epsilon / \partial k_{z_{j}}^{2}\right\rangle$ in the limit of a weakly interacting gas and inserting the results for $m_{1}$ and $m_{-1}$ into Eq.(22), one recovers result (21) for the frequency of the center of mass oscillation.

Equations (20), (21) hold for any value of the laser intensity provided the system remains superfluid. In the tight binding limit the formalism becomes particularly simple. In fact in this case the effective mass (20), can be conveniently written as $1 / m_{C M}=f / m^{*}$, where

$$
f=\frac{5}{2} \frac{\int h(x)^{3 / 2} \sin ^{2} x \Theta[h(x)] d x}{\int h(x)^{5 / 2} \Theta[h(x)] d x}
$$

is a dimensionless function of the ratio $\epsilon_{F} / 2 \delta$ and the dependence of $m^{*}=\hbar^{2} / \delta d^{2}$ on the laser intensity $s$ is given, for example, in Ref. [5] . The function $h(x)$ is defined by $h(x)=\epsilon_{F} / \delta-1+\cos x$. Here $\epsilon_{F}$ is the Fermi energy which is related to the free value $\epsilon_{F}^{0}=(3 N)^{1 / 3} \hbar\left(\omega_{\perp}{ }^{2} \omega_{z}\right)^{1 / 3}$ evaluated in the absence of the periodic potential, by the equation

$$
\left(\epsilon_{F}^{0}\right)^{3}=\frac{16}{5 \pi^{2}}\left(\frac{E_{R}}{\delta}\right)^{1 / 2} \delta^{3} \int_{-\pi}^{\pi} h(x)^{5 / 2} \Theta[h(x)] d x,
$$

which follows from the normalization condition $N=$ $\int n_{0}(\mathbf{r}) d \mathbf{r}$. 
In Fig 1 we plot $f$ as a function of the parameter $y=$ $\epsilon_{F} / 2 \delta$. In the limit $\epsilon_{F} \ll 2 \delta, \tilde{m}$ does not depend on the density and one finds $f=1$ and $m_{C M}=m^{*}=\hbar^{2} / \delta d^{2}$. For higher values of the density, the frequency of the oscillation becomes smaller because $m_{C M}$ increases with the density. In the limit $\epsilon_{F} \gg 2 \delta$, we find the asymptotic behavior $f=5 \delta / 4 \epsilon_{F}$ (see dashed line in Fig 1) and $m_{C M}=4 \hbar^{2} \epsilon_{F} / 5 d^{2} \delta^{2}$.

As an example, we consider a two-component gas of $N=10^{5}$ potassium $\left({ }^{40} \mathrm{~K}\right)$ atoms with trap frequencies $\omega_{\perp}=2 \pi \cdot 275 \mathrm{~Hz}$ and $\omega_{z}=2 \pi \cdot 24 \mathrm{~Hz}$, corresponding to $\epsilon_{F}^{0} / k_{B}=390 \mathrm{nK}$. For the optical lattice we assume $s=5$ and periodicity $d=400 \mathrm{~nm}$, corresponding to $E_{R}=$ $9.2 \delta=374 n K$. From Eq. (24) one finds $\epsilon_{F}=0.85 \epsilon_{F}^{0}$ and from the value $\epsilon_{F} / 2 \delta=4.1$, one finds $f(4.1)=0.17$, yielding $m_{C M} / m=10.94$. Eq.(21) predicts a significant reduction $\left(\omega_{C M}=0.30 \omega_{z}\right)$ of the frequency of the center of mass oscillation. Notice indeed that this reduction is larger than in the corresponding case of an oscillating Bose-Einstein condensate, where $\omega_{C M}=\sqrt{m / m^{*}} \omega_{z}=$ $0.73 \omega_{z}$.

In conclusion we have investigated the dynamic behaviour of a superfluid Fermi gas in the presence of a 1D optical lattice. We have calculated the velocity of sound and studied the conditions of stability (both energetical and dynamical) against the creation of elementary excitations when the fluid moves with respect to the lattice. Moreover, we have calculated the frequency of the center of mass oscillation (in the BCS limit) when the gas is further confined by a harmonic trap. Our results might be useful for the identification of the superfluid phase of interacting Fermi gases. Near a Feshbach resonance, where the scattering length becomes very large, one still expects the sytem to remain superfluid for moderate intensities of the laser field and, consequently, to exhibit undamped center of mass oscillations in the presence of harmonic trapping. In this case, however, the evaluation of the effective mass cannot be based on Eqs. (1020) and requires a more complete many-body calculation.

This work was supported by the Ministero dell'Istruzione, dell'Universita' e della Ricerca
(M.I.U.R.).

[1] J. Javanainen, Phys. Rev. A, 60, 4902 (1999). See also C. Menotti et al., cond-mat/0404272 [Phys. Rev. A (to be published)] and references therein.

[2] F.S. Cataliotti, S. Burger, C. Fort, P. Maddaloni, F. Minardi, A. Trombettoni, A. Smerzi, M. Inguscio, Science 293, 843 (2001); C. Fort, F.S. Cataliotti, L. Fallani, F. Ferlaino, P. Maddaloni and M. Inguscio, Phys. Rev. Lett. 90140405 (2003)

[3] M. Kramer, L.P. Pitaevskii and S. Stringari, Phys. Rev. Lett. 88, 180404 (2002)

[4] Since in a weakly interacting gas the Fermi energy is much larger than the superfluid gap, the momentum distribution in the superfluid differs very little from the noninteracting value $n_{k}$.

[5] L.P. Pitaevskii and S. Stringari, "Bose-Einstein Condensation", Oxford University Press (2003)

[6] J.R. Schrieffer, Theory of Superconductivity, AddisonWesley, New York (1971)

[7] P.W. Anderson, Phys. Rev. 112, 1900 (1958); see also M. Randeria in "Bose-Einstein Condensation", Cambridge University Press (1995)

[8] Y. Tanaka, Physica C 219 213-220 (1994)

[9] C. Menotti, A. Smerzi and A. Trombettoni, New J. Phys. 5, $112(2003)$

[10] L. Fallani et al., Phys. Rev. Lett. 93, 140406 (2004)

[11] In the case of Bose condensates a generalization of the above results to arbitrary values of $q$ can be obtained in the framework of the Gross-Pitaevskii theory, see Ref. 9].

[12] L. Pezzè et al., Phys. Rev. Lett. 93, 120401 (2004)

[13] G. Orso, L.P. Pitaevskii and S. Stringari, Phys. Rev. Lett. 93020404 (2004)

[14] M. Wouters, J. Tempere and J.T. Devreese, cond-mat/0312154 [Phys. Rev. A (to be published)]

[15] Notice that result (20) for the effective mass of the superfluid trapped gas differs from the result $m_{C M}=$ $(2 / N) \int k_{z}^{2} \delta\left(\epsilon_{F}(\mathbf{r})-\epsilon(\mathbf{k})\right) d \mathbf{k} d \mathbf{r} /(2 \pi \hbar)^{3}$ holding in the collisional regime of a degenerate Fermi gas [13]. The two results coincide only if the dispersion $\epsilon\left(k_{z}\right)$ is quadratic.

[16] D. Pines and P. Nozieres, The theory of quantum liquids, Addison-Wesley, New York, 1989. Oxford University 